\title{
Evaluating the Impact of Strategic Personnel Policies Using a MILP Model: The Public University Case
}

\author{
Rocio de la Torre (iD), Amaia Lusa (iD), Manuel Mateo ${ }^{(\mathbb{D}}$ \\ Department of Management, Universitat Politècnica de Catalunya (UPC) (Spain) \\ maria.rocio.de.torre@upc.edu, amaia.lusa@upc.edu, manel.mateo@upc.edu
}

Received: July 2016

Accepted: October 2016

\section{Abstract:}

Purpose: The main purpose of the paper is to evaluate the impact of diverse personnel policies around personnel promotion in the design of the strategic staff plan for a public university. The strategic staff planning consists in the determination of the size and composition of the workforce for an organization.

Design/methodology/approach: The staff planning is solved using a Mixed Integer Linear Programming (MILP) model. The MILP model represents the organizational structure of the university, the personnel categories and capacity decisions, the demand requirements, the required service level and budget restrictions. All these aspects are translated into a set of data, as well as the parameters and constraints building up the mathematical model for optimization. The required data for the model is adopted from a Spanish public university.

Findings: The development of appropriate policies for personnel promotion can effectively reduce the number of dismissals while proposing a transition towards different preferable workforce structures in the university.

Research limitations/implications: The long term staff plan for the university is solved by the MILP model considering a time horizon of 8 years. For this time horizon, the required input data is derived from current data of the university. Different scenarios are proposed considering 
different temporal trends for input data, such as in demand and admissible promotional ratios for workers.

Originality/value: The literature review reports a lack of formalized procedures for staff planning in universities taking into account, at the same time, the regulations on hiring, dismissals, promotions and the workforce heterogeneity, all considered to optimize workforce size and composition addressing not only an economic criteria, but also the required workforce expertise and the quality in the service offered. This paper adopts a formalized procedure developed by the authors in previous works, and exploits it to assess the impact of various personnel policies in the staff planning for a particular university case, and this is the principal contribution of the paper.

Keywords: strategic staff decisions, university, MILP

\section{Introduction}

During the last decades, in many European countries, the Higher Education Institutions (HEIs) have been facing new pressures in an increasingly business oriented environment: the reformulation and unification of academic degrees in Europe; the creation of the European Research Area (ERA); the identification of new public funding; the increasing emphasis on university-industry relationship; and the stress in patenting and entrepreneurship (Mckelvey \& Holmen, 2009). Altogether impose great requirements for organizations to develop new strategies and reaction protocols.

Consequently, HEIs have been adopting various management strategies in the last years. These strategies aim to determine in a long term horizon, the quantity and type of resources for the organization, considering the economic criteria, apart from other possible aspects of different nature. Since the main resource in HEIs is the workforce, the strategic staff plan becomes a key element in the design and sustainability of the universities. Some authors (Clark, 2003; Agasisti, Arnaboldi \& Azzone, 2008) indicate that the number of strategic planning practices in HEIs is increasing and diversifying. Yet, as pointed out by Machuca, González-Zamora and Aguilar-Escobar (2007), there has been a gap between the growing importance in the strategic planning for the organization of HEIs, and public services in general, and the number of studies in literature. Moreover, these practices, when supported by formalized procedures, are so far adopted by manufacturing industry, noticing very few examples in the service sector (i.e. call centres, supermarkets and so on). For HEIs, adopting tools for assessing the strategic staff planning it is particularly convenient taking into account diverse factors such as: the rigid regulations in the university policies, the low availability in the labour market of suitable candidates and the very long learning periods 
required for workers, among others. The development of a formalized procedure for the strategic staff planning in public universities was previously addressed by the authors in de la Torre, Lusa and Mateo $(2014,2016)$. These works report the lack of formalized procedures for staff planning in universities taking into account, at the same time, the regulations on hiring, dismissals, promotions and the workforce heterogeneity, all considered to optimize workforce size and composition addressing not only an economic criteria, but also the required workforce expertise and the service level offered. The development of a formalized procedure for the staff planning considering the above mentioned aspects was precisely the main contribution of the cited works. Complementarily, this paper exploits the developed procedure to assess the impact of various personnel policies in the staff planning for a particular university case.

The paper is organized as follows: Section 2 describes the problem and details the scope of the article; Section 3 summarizes the MILP model previously developed by the authors, for solving the staff planning; Section 4 introduces the computational scenarios, which are object of analysis. Finally, the conclusions and further research are detailed in Section 5.

\section{Problem Description and Scope of the Article}

Several aspects characterizing the university are addressed in the present paper so as to solve the strategic staff plan: the organizational structure, the personnel categories and capacity decisions, the teaching capacity requirements (demand), the required service level and finance-related aspects. These aspects are detailed in previous works: de la Torre et al. $(2014,2016)$. All of them are translated into a set of data, parameters and constraints, all defining a mathematical model for optimization (see Section 3). Although already defined, some of the principal characteristics of the problem are detailed in the following for the sake of clarity (de la Torre et al., 2016):

- A worker can belong just to one unit or department. The category determines the capacity of a worker.

- There are two types of categories: temporary and permanent ones. Workers in temporary categories should progress to a top category after a certain period of time to keep working in the university. Conversely, workers in permanent categories are able to, but it is not mandatory to progress in the organization architecture.

- The considered personnel decisions in the strategic staff planning process are workforce hiring, promotion and dismissal.

- There is a maximum budget for personnel costs. 
- The evaluation criteria for the development of the staff plan comprises the personnel costs and the achievement of a preferable composition for the academic staff.

- Forecasted layoffs and teaching hours for each department are known for the considered time horizon for staff planning.

- Finally, the academic pathway for workers is known (see Figure 1).

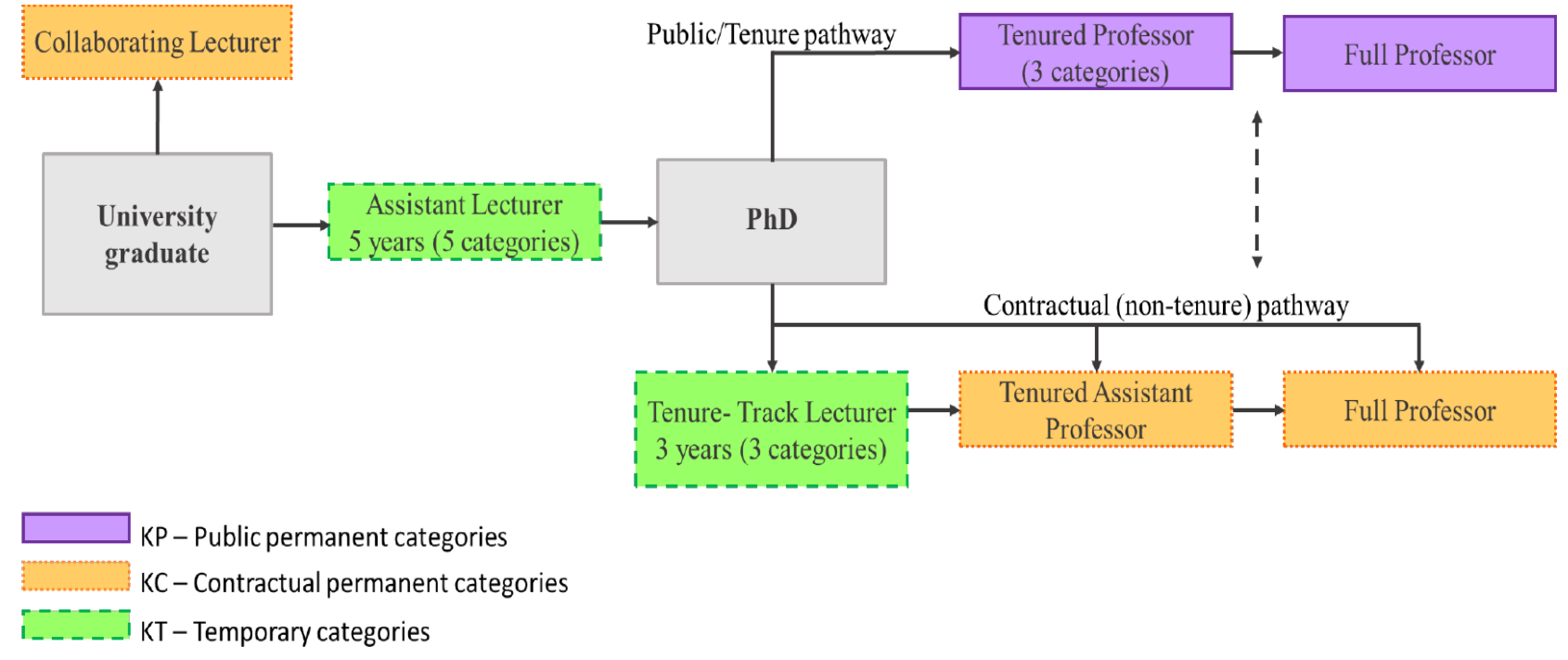

Figure 1. Evolution of the academic career (de la Torre et al., 2016)

The evaluation criteria for the optimization problem, as previously noted in the introduction, is two-fold: i) to minimize personnel costs; and ii) to determine a workforce pyramid according to a preferable one, or university model. In regard of the first evaluation criteria, this paper considers diverse personnel expenditures such as salaries, hiring and dismissals. Adopting the second evaluation criteria, the determined workforce composition considers people from each subset of categories, i.e. the part time staff, the permanent staff, the contracted staff. Conversely, the program will determine a heterogeneous staff composition, thus favoring the quality in the service provided and the development of other tasks apart from teaching, as research and technology transfer. Indeed, by doing this, one university may intensify efforts in research and technology transfer adopting a workforce composition with an important share in $\mathrm{PhD}$ students and professor assistants. This way modulating the size of workforce held by $\mathrm{PhD}$ students and experienced professors one can also modulate the volume of research and technology transfer carried out in the university. In general terms, we can state that the selection of different workforce pyramids would respond to different strategic visions for the university. 
The economic optimization of the staff plan and the achievement of a preferable workforce composition are affected by personnel policies such as those related to personnel promotion. For instance, low admissible promotion ratios can affect internal mobility of workers and the university will need other mechanisms to achieve the preferable workforce composition. Further, the achievement of the preferable composition can be also bounded by prioritizing or not internal promotions, as well as by permitting or not the dismissal of permanent workers. The impacts that these strategic policies have in the long term staff plan are object of study in this paper.

\section{Modelling}

The problem has been modelled by using MILP, based on de la Torre et al. $(2014,2016)$. The readers are referred to these works for a detailed description of the model. The set of units composing the university is noted by $U$, and the set of periods (or years) for optimization purposes is T. Finally, the number of categories building up workforce is $K$. As explained further in Section 4, the set of categories is divided into three subsets: a subset comprising temporary categories, $K T$; a subset concerning permanent categories following a tenure pathway KP; and a subset concerning also permanent categories, but following a non-tenure pathway $K C$. For the sake of clarity though, few unavoidable notes are included in the following. The objective function is formulated by

$$
\begin{aligned}
{[M I N] z } & =\underbrace{\sum_{u \in U, t \in T}\left[\sum_{k \in K}\left(C W_{k t} \cdot w_{u k t}\right)+C A_{t} \cdot a_{u t}\right]+C F \cdot\left[\sum_{u \in U, k \in K, t \in T} w_{u k t}^{-}\right]}_{\text {part } i)}+ \\
& +\underbrace{\sum_{u \in U, k \in K, t \in T}\left[\lambda_{k t} \cdot\left(d_{u k t}^{+}+d_{u k t}^{-}\right)\right]+\sum_{u \in U, t \in T}\left(\mu_{t} \cdot d_{u t}\right)+\omega \cdot \sum_{t \in T} d_{t}}_{\text {part ii })}
\end{aligned}
$$

As it can be noted, the function is composed by two main parts. The part i) represents the personnel costs, while the part ii) is associated to the discrepancies between preferable and planned workforce composition. Personnel costs are proportional to the number of full time workers $w_{u k t}$ per unit $u$, category $k$ and period $t$, the number of part time workers $a_{u t}$ and the dismissals $w_{u k t}^{-}$. These are decision variables for the problem, while $C w_{k t}, C A_{t}$ and $C F$ are costs in monetary units per worker, so input data for the problem.

The parameters in the part ii) are derived from the sum of positive and negative discrepancies $d_{u k t}^{+}$and $d_{u k t}^{-}$, respectively, between the preferable and the planned composition for each unit, category and period. In addition, from the maximum value of $d_{u k t}^{+}$and $d_{u k t}^{-}$, the variable $d_{u t}$ is defined. Further, $d_{t}$ is determined as the maximum of all $d_{u l}$. These last two variables are included to avoid insofar as possible 
that discrepancies concentrate on particular categories and periods, respectively. To translate each of the above described three terms weighting workforce discrepancies, $\left(d_{u k t}^{+}+d_{u k t}^{-}\right), d_{u t}$ and $d_{t}$, the economic penalty coefficients $\lambda_{k t}, \mu_{t}$ and $\omega$ are included. For the purposes of the paper, these are input data defined, respectively, as the annual salary per each category and worker; as a proportion of the annual average budget for a department or unit; and as a proportion of the annual budget for the entire university.

The MILP problem is subjected to several constraints depicting the rules for workforce evolution and towards a preferable composition. All these constraints can be consulted in de la Torre et al. (2016). However, and since directly related to the object of the study in this paper, it is important to explicitly address here a set of constraints defined around personnel promotion. In order to represent policies prioritizing personnel promotion in the model, a binary variable $y_{\text {usk t }}$ is defined. This is an auxiliary variable for prioritizing the promotion of the current workers from the category $s$ to the category $k$ over hiring workers from the labor market for the category k. The inclusion of $y_{u s k t}$ is mathematically stated as

$$
y_{u s k t} \in\{0,1\} \quad u \in U, s \in K \mid K_{s}^{+} \neq\{\phi\}, k \in K_{s}^{+}, t \in T
$$

Where $K_{s}^{+}$is the set of categories to which is possible to access from the category s. Using $y_{u s k \text {, }}$ the number of promotions $q_{u s k t}$ and workers hired from the labor market $w_{u k t}^{+}$are bounded by the following constraints,

$$
\begin{array}{ll}
q_{u s k t} \geq R_{u s k t} \cdot w_{u k, t-1}-R_{u s k t} \cdot M \cdot y_{u s k t}-1 & u \in U, s \in K \mid K_{s}^{+} \neq\{\phi\}, k \in K_{s}^{+} \\
w_{u k t}^{+} \leq M \cdot\left(1-y_{u s k t}\right) & u \in U, s \in K \mid K_{s}^{+} \neq\{\phi\}, k \in K_{s}^{+}
\end{array}
$$

Where $R_{\text {us } k \text { t }}$ is the proportion of workers (established by the own university) that can promote, as maximum, from the category $s$ to the category $k$ in the unit $u$ and period $t ; C_{u t}$ is the required teaching hours for the unit $u$ and period $t ; E C_{u t}$ is the excess of capacity that should have, at least, the unit $u$ in the period $t ; H_{k t}$ is the capacity in hours associated to each worker in the category $k$ in the period $t$; and $M=C_{u t} \cdot\left(1+E C_{u t}\right) / H_{k t}$. All the above magnitudes are input data for the model. Equations (3) and (4) force $w_{u k t}^{+}$equal to zero provided that the number of workers promoted to a category $k$ does not exceed the upper bound defined by $M$. 


\section{Scenarios}

Diverse strategic visions for the future in a university can be translated into different workforce structure. To establish the preferable structures, a poll on university management was addressed to a group of relevant academics. The results yielded three preferable compositions:

Model A. The university is devoted to create knowledge to be exported to other sectors. This way, an academic structure based on the training of a huge volume of assistant professors and $\mathrm{PhD}$ students can be defined. This yields a workforce composition with an important share in personnel within subset KT. This subset presents high rotation rates and reduced capacity.

Model B. Here the pursued size of workforce in $K T$ is sensibly lower than in the model A. The number of workers under $K T$ is adequate just to address the generational replacement of the university, therefore retaining the generated knowledge. Accordingly, mentoring programs for $\mathrm{PhD}$ students and professor assistants are developed.

Model C. This model C proposes to configure a workforce with high expertise. These workers build commonly subsets $K P$ and $K C$. The vision here is according to the idea that experienced academics can develop more tasks and with better performance than those executed by less experienced ones. One potential drawback of this model is the advisable scarcity of young researchers in $K T$. Therefore, the generational replacement could be compromised and/or satisfied by an important share of workers hired from the labor market.

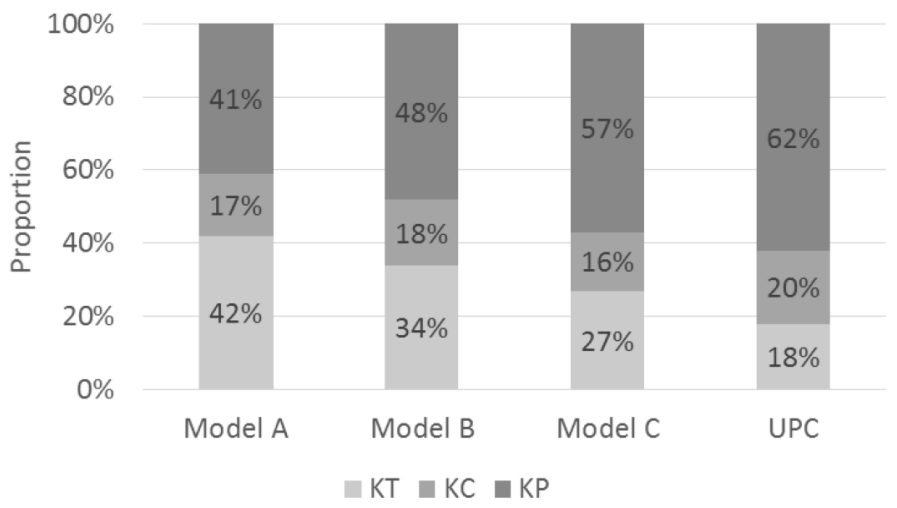

Figure 2. Preferable workforce compositions: university models, A, B and C. The last column in the right side presents the current workforce composition of the UPC (with 1999 workers)

The results of the above-mentioned poll are presented in Figure 2. It is worth noting that for all university models, the preferable weight of categories within $K C$ is almost the same. The last column 
in the right side presents the current workforce composition of the Universitat Politècnica de Catalunya (UPC), a public university in Spain. Their data is adopted in this paper as a case study (see Section 5).

The adoption of the university models $\mathrm{A}$ to $\mathrm{C}$, along with different temporal trends for promotion ratios and personnel budget, yields different computational scenarios, all answering the objectives of the present paper. The computational scenarios are listed in Table 1 and graphically depicted in Figure 3. By proposing a two-step analysis evaluating separately scenarios 1 to 3 and 4 to 6 , one can discuss on the impact of admissible promotion ratios in the staff plan, also considering the options of dismissing workers in $K C$ as well as the decision of prioritizing internal promotions.

\begin{tabular}{|c|c|c|c|}
\hline Scenario & Adm. Promotion ratio & Dismissals in $\boldsymbol{K} C$ & Promotions prioritized \\
\hline 1A / 1B / 1C & Constant & Yes & No \\
\hline 2A / 2B / 2C & Increasing & Yes & No \\
\hline 3A / 3B / 3C & Decreasing & Yes & Yes \\
\hline $4 \mathrm{~A} / 4 \mathrm{~B} / 4 \mathrm{C}$ & Constant & No & Yes \\
\hline 5A / 5B / 5C & Increasing & No & Yes \\
\hline 6A $/ 6 \mathrm{~B} / 6 \mathrm{C}$ & Decreasing & No & \\
\hline
\end{tabular}

Table 1. List of computational scenarios

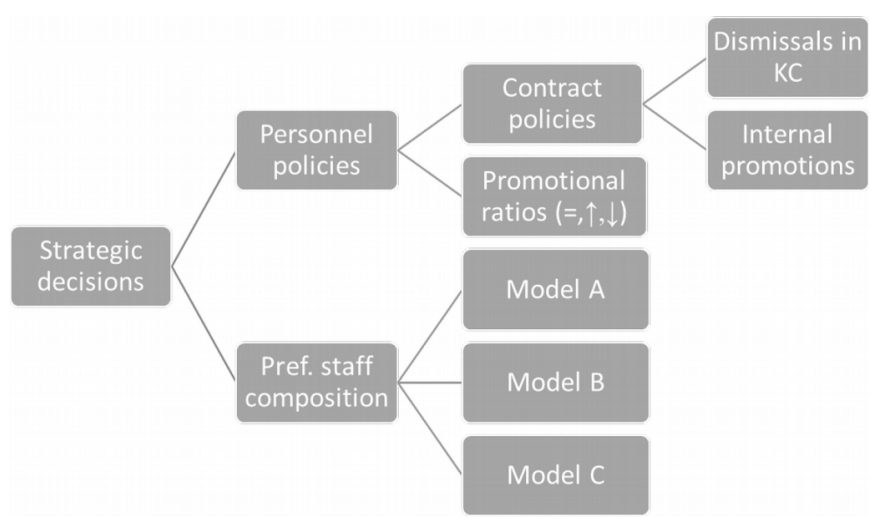

Figure 3. Graphical explanation of computational scenarios 


\section{Analysis of the Results}

As previously introduced, the proposed case study in this paper is based on data from the UPC. Its portfolio comprises 68 degrees and masters, mainly in the field of engineering, hosting around 30000 students in 23 schools and faculties. At the time of this research, the total workforce was around 3000 people in 42 units $(u=42)$ or departments. The academic workforce, which is the considered personnel for optimization purposes in this paper, reaches 1999 people. Further, workers are organized in $k=15$ categories. Eight of the total number of categories are temporary ones (one category for each of the 8 years a worker can be with temporary contracts), 4 are permanent under the subset $K P$, and 3 are under the subset KC, UPC (2014).

As depicted in Figure 2, given the contract policies for the UPC in the last years, the current workforce structure is closer to model $\mathrm{C}$ than to the rest. It is remarkable the little amount of workers in $K T$ (just $18 \%$ of total workers); thus, permanent contracts for experienced workers with high capacity are preferred.

The MILP model for strategic staff planning adopts the above described initial workforce composition for the UPC. The results of the model under the conditions of scenarios 1 to 3 are summarized in Table 2. As it can be observed, decreasing trends in $R_{\text {uskt }}$ greatly impacts the strategic staff plan, since worker's promotions decrease substantially (see results for scenario 3, compared to those for scenario 1). This reduction in worker's promotions is also accompanied by an incremental number of dismissals. This way, the possibility of dismissing workers is adopted as a mechanism towards the achievement of the preferable workforce.

\begin{tabular}{|c|r|r|r|r|r|r|r|r|r|}
\hline \multirow{2}{*}{ Metric } & \multicolumn{3}{|c|}{$\begin{array}{c}\text { Scenario 1 } \\
\text { (const. promotion ratio) }\end{array}$} & \multicolumn{3}{c|}{$\begin{array}{c}\text { Scenario 2 } \\
\text { (incr. promotion ratio) }\end{array}$} & \multicolumn{3}{c|}{$\begin{array}{c}\text { Scenario 3 } \\
\text { (decr. promotion ratio) }\end{array}$} \\
\cline { 2 - 11 } & Mod. A & Mod. B & Mod. C & Mod. A & Mod. B & Mod. C & Mod. A & Mod. B & Mod. C \\
\hline Promotions & 921 & 849 & 672 & $+0.3 \%$ & $+3.7 \%$ & $+1.2 \%$ & $-9.5 \%$ & $-11.4 \%$ & $-12.8 \%$ \\
\hline Dismissals & 129 & 132 & 160 & $-0.7 \%$ & $-0.6 \%$ & $+1.5 \%$ & +11.6 & $+8.7 \%$ & $+11.3 \%$ \\
\hline Workforce size $(t=8)$ & 1599 & 1533 & 1443 & $-0.4 \%$ & $+0.2 \%$ & $-0.7 \%$ & $-0.8 \%$ & $-0.9 \%$ & $-2.1 \%$ \\
\hline
\end{tabular}

Table 2. Impact assessment of considering different admissible promotion ratios. Dismissals for workers in $K C$ are permitted. $R_{u s k t}$ monotonically varies by $+5 \%$ per year (scenario 2 ) and $-5 \%$ per year (scenario 3 ).

Results for scenarios 2 and 3 are relative to those for scenario 1

Figure 4 relates the total number of promotions and the dismissals for workers in $K C$, with different increasing and decreasing yearly variations in $R_{u s k t}$ of up to $\pm 15 \%$. Results are presented as relative to scenario 1 , which is characterized by comprising constant admissible promotion ratio throughout the time 
horizon. The upper graph clearly depicts a correlation between the considered trend (either positive or negative) for $R_{u s k t}$ and the number of promotions. However, such correlation is not clear for dismissals in case of considering positive trends for $R_{u s k \text {. }}$

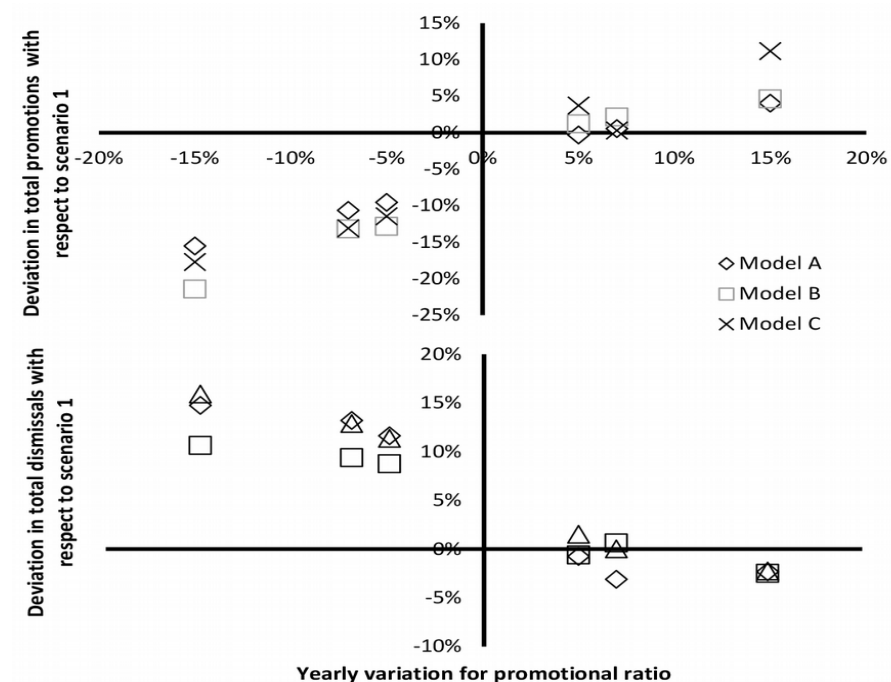

Figure 4. Relationship between temporal trends in admissible promotion ratio with dismissals for workers in $K C$ and total promotions. Note that positive temporal trends for $\mathrm{R}_{u s k t}$ correspond to scenario 2 , while negative ones correspond to scenario 3

The above analysis concerns dismissals for workers in $K C$. The same discussion can be proposed now considering scenarios 4 to 6 , so forbidding dismissals (see Table 3). Considering together the data in Tables 2 and 3, it can be concluded that the application of a sustained decrement in $R_{u s k t}$ is translated into a reduction in the number of internal promotions, regardless dismissals for workers in $K C$ are permitted or not. This reduction in the number of promotions is sensibly higher in the case of dismissals for workers in $K C$ are forbidden.

\begin{tabular}{|c|r|r|r|r|r|r|r|r|r|}
\hline \multirow{2}{*}{ Metric } & \multicolumn{2}{|c|}{$\begin{array}{c}\text { Scenario 4 } \\
\text { (const. budget and } \\
\text { promotion ratio) }\end{array}$} & \multicolumn{2}{c|}{$\begin{array}{c}\text { Scenario 5 } \\
\text { (incr. budget and } \\
\text { promotion ratio) }\end{array}$} & \multicolumn{3}{c|}{$\begin{array}{c}\text { Scenario 6 } \\
\text { (decr. budget and } \\
\text { promotion ratio) }\end{array}$} \\
\cline { 2 - 11 } & Mod. A & Mod. B & Mod. C & Mod. A & Mod. B & Mod. C & Mod. A & Mod. B & Mod. C \\
\hline Promotions & 1521 & 1447 & 1412 & $+5.8 \%$ & $+3.7 \%$ & $+3.9 \%$ & $-11.6 \%$ & $-12.3 \%$ & $-15.2 \%$ \\
\hline Workforce size $(t=8)$ & 1594 & 1531 & 1436 & $+0.5 \%$ & $+0.6 \%$ & $+0.4 \%$ & $-0.5 \%$ & $-0.3 \%$ & $-0.3 \%$ \\
\hline
\end{tabular}

Table 3. Impact assessment of considering different admissible promotion ratios. Dismissals for workers in $K C$ are not permitted. $R_{\text {usket }}$ monotonically varies by $+5 \%$ per year (scenario 5 ) and $-5 \%$ per year (scenario 6 ).

Results for scenarios 5 and 6 are relative to those for scenario 4 


\section{Conclusions and Further Research}

The model includes the most relevant issues of long term staff planning at public universities (since public universities are usually more flexible). Furthermore, the model gives optimal or near-optimal solutions in reasonable times and the quality of the solutions is good. The optimization model is a useful tool that permits to determine the optimum size and composition of the workforce in a long term horizon and has enough flexibility to give good solutions even if more constraints are added (e.g., allowing or not dismissals in $K C$ or prioritizing or not internal promotions). Also, the optimization model permits to easily define various computational scenarios as a strategic planning tool, from which evaluate the impact of strategic policies before implementing them into the organization. The main applications of this planning tool are: to update the plan for workforce and the assessment of the impact that different strategies may have on the personnel costs and the structure of a university; i.e. the accomplishment of a preferable staff composition, adding/eliminating new courses or studies; increasing/reducing the number of students per group; changes in teaching capacity requirements; investment in training and research; changes in the proportion of people that can be promoted; allowing or not dismissals in non-tenure track staff; or prioritizing promotions over external hiring. The main conclusion of the paper is that personnel policies directly impact the economic optimization -and towards a preferable workforce composition- of the long term staff plan in universities. The development of adequate policies around personnel promotion can reduce the number of workers dismissed while proposing a transition towards a different preferable workforce structure based on the promotional ratios. The formalized procedure adopted in this paper (based on a MILP model) is adequate to address different aspects around the strategic staff plan.

Further, some research lines can be derived from this paper. The first of them could be to consider promotional ratio as a decision variable for the problem, all function of the investment in research and training. Secondly, the model could be improved to consider the uncertainty in some input data (demand, promotional ratios, among others). Thirdly, research could be focused on the application of the optimization problem for the staff planning of other KIOs organizations such as business consultancy.

\section{Acknowledges}

Supported by DPI2015-67740-P (MINECO/FEDER) project. 


\section{References}

Agasisti, T., Arnaboldi, M., \& Azzone, G. (2008). Strategic management accounting in universities: the Italian experience. Higher Education, 55(1), 1-15. https://doi.org/10.1007/s10734-006-9032-6

Clark, B.R. (2003). Sustaining change in universities: continuities in case studies and concepts. Tertiary Education and Management, 9, 99-116. https://doi.org/10.1080/13583883.2003.9967096

De la Torre, R., Lusa, A., \& Mateo, M. (2014). Methodology for the strategic capacity planning in universities. Managing Complexity: Challenges for Industrial and Operations Management. Springer, 333-340. https://doi.org/10.1007/978-3-319-04705-8_39

De la Torre, R., Lusa, A., \& Mateo, M. (2016). A MILP model for the long term academic staff size and composition planning in public universities. Omega, The International Journal of Management Science, 63, 1-11. https://doi.org/10.1016/j.omega.2015.09.008

Machuca, J.A.D., González-Zamora, M.M., \& Aguilar-Escobar, V.G. (2007). Service operations management research. Journal of Operations Management, 25, 585-603. https://doi.org/10.1016/j.jom.2006.04.005

Mckelvet, M., \& Holmen, M. (2009). Learning to compete in European universities: from social institution to knowledge business. Edward Elgar Publishing Limited, Cheltenham. https://doi.org/10.4337/9781848446106

UPC (Universitat Politècnica de Catalunya BarcelonaTech) (2014). http://www.upc.edu (Accessed: September 2014).

Journal of Industrial Engineering and Management, 2016 (www.jiem.org)

Article's contents are provided on an Attribution-Non Commercial 3.0 Creative commons license. Readers are allowed to copy, distribute and communicate article's contents, provided the author's and Journal of Industrial Engineering and Management's names are included. It must not be used for commercial purposes. To see the complete license contents, please visit http://creativecommons.org/licenses/by-nc/3.0/. 